\title{
SPIDERS' WEBS IN THE PUNCTURED PLANE
}

\author{
VASILIKI EVDORIDOU, DAVID MARTÍ-PETE, AND DAVID J. SIXSMITH
}

This paper is dedicated to our doctoral supervisors Gwyneth Stallard and Phil Rippon, with thanks for all their help and support

\begin{abstract}
Many authors have studied sets, associated with the dynamics of a transcendental entire function, which have the topological property of being a spider's web. In this paper we adapt the definition of a spider's web to the punctured plane. We give several characterisations of this topological structure, and study the connection with the usual spider's web in $\mathbb{C}$.

We show that there are many transcendental self-maps of $\mathbb{C}^{*}$ for which the Julia set is such a spider's web, and we construct a transcendental self-map of $\mathbb{C}^{*}$ for which the escaping set $I(f)$ has this structure and hence is connected. By way of contrast with transcendental entire functions, we conjecture that there is no transcendental self-map of $\mathbb{C}^{*}$ for which the fast escaping set $A(f)$ is such a spider's web.
\end{abstract}

\section{INTRODUCTION}

Let $S$ be either the complex plane $\mathbb{C}$ or the punctured plane $\mathbb{C}^{*}:=\mathbb{C} \backslash\{0\}$, and suppose that $f: S \rightarrow S$ is a holomorphic function such that $\widehat{\mathbb{C}} \backslash S$ consists of essential singularities of $f$, where $\widehat{\mathbb{C}}:=\mathbb{C} \cup\{\infty\}$. We define the Fatou set of $f$ by

$F(f):=\left\{z \in S:\left\{f^{n}\right\}_{n \in \mathbb{N}}\right.$ is a normal family in an open neighbourhood of $\left.z\right\}$, and we let the Julia set be its complement in $S$; that is, $J(f):=S \backslash F(f)$. We use the term Fatou component to refer to each component of $F(f)$.

When $S=\mathbb{C}, f$ is a transcendental entire function. There is a long history of the study of the dynamics of these functions dating back to the 1920s; see [Ber93] for a general reference. When $S=\mathbb{C}^{*}$, we say that $f$ is a transcendental self-map of $\mathbb{C}^{*}$. The study of the dynamics of these maps dates back to Rådström [Råd53], and many authors have added to this subsequently; see, for example, [Bak87, Kee88, Kot87, Mak87, Mak91]. This paper concerns the iteration of this second class of functions, but we begin by reviewing some results for the first class of functions by way of comparison.

The first author was supported by Engineering and Physical Sciences Research Council grant $\mathrm{EP} / \mathrm{R} 010560 / 1$. The second author was supported by the grant-in-aid 16F16807 from the Japan Society for the Promotion of Science.

2010 Mathematics Subject Classification. Primary 37F10; Secondary 30D05.

Key words: holomorphic dynamics, escaping set, punctured plane, spider's web. 
For a transcendental entire function $f$, the escaping set of $f$ is defined by

$$
I(f):=\left\{z \in \mathbb{C}: f^{n}(z) \rightarrow \infty \text { as } n \rightarrow \infty\right\} .
$$

Eremenko Ere89 showed that all components of $\overline{I(f)}$ are unbounded, and conjectured that, in fact, all components of $I(f)$ are unbounded. This conjecture, known as Eremenko's conjecture, is still open and has motivated much research in transcendental dynamics in the past 30 years.

It is clear that Eremenko's conjecture holds in a strong way when $I(f)$ is connected. Rippon and Stallard [RS12] studied several classes of transcendental entire functions for which this is true, and introduced the topological notion of a spider's web. This is defined as follows.

Definition 1.1 (Spider's web). A set $E \subset \mathbb{C}$ is a spider's web if $E$ is connected, and there exists a sequence $\left(G_{n}\right)_{n \in \mathbb{N}}$ of bounded simply connected domains such that

$$
\partial G_{n} \subset E, G_{n} \subset G_{n+1}, \text { for } n \in \mathbb{N}, \quad \text { and } \bigcup_{n \in \mathbb{N}} G_{n}=\mathbb{C} \text {. }
$$

Roughly speaking, a set is a spider's web if it is connected, and it contains a sequence of "loops" surrounding each other that tend to infinity. Rippon and Stallard showed that there are many classes of transcendental entire functions for which $I(f)$ is a spider's web, and so Eremenko's conjecture holds for these functions.

In fact, Rippon and Stallard studied the following two subsets of the escaping set. Suppose that $f$ is a transcendental entire function. First we define the maximum modulus function

$$
M(r):=M(r, f):=\max _{|z|=r}|f(z)|, \quad \text { for } r \geq 0 .
$$

Choose $R>0$ sufficiently large that $M^{n}(R) \rightarrow+\infty$ as $n \rightarrow \infty$. We then define the level set

$$
A_{R}(f):=\left\{z \in \mathbb{C}:\left|f^{n}(z)\right| \geq M^{n}(R) \text { for all } n \geq 0\right\},
$$

and the fast escaping set

$$
A(f):=\bigcup_{\ell \geq 0} f^{-\ell}\left(A_{R}(f)\right)
$$

It can be shown that the definition of $A(f)$ is independent of the choice of $R$. Note that $A(f)$ is completely invariant under $f$, that is, $f^{-1}(A(f))=A(f)$, but $A_{R}(f)$ is not.

In $\left[\right.$ RS09 it was shown that if $A_{R}(f)$ is a spider's web, then so is $A(f)$, and also that if $A(f)$ is a spider's web, then so is $I(f)$. Rippon and Stallard gave a number of conditions each of which implies that $A_{R}(f)$ is a spider's web and so, in particular, $I(f)$ is connected; see RS12, Theorem 1.9], and also see [Six11 for many other examples. They also asked the following questions.

Question 1. Is there a transcendental entire function $f$ such that $A(f)$ is a spider's web but $A_{R}(f)$ is not?

Question 2. Is there a transcendental entire function $f$ such that $I(f)$ is a spider's web but $A(f)$ is not? 
Question 1 is still open. Question 2 was first answered in the positive by a complicated example in [RS13. Later Evdoridou [Evd16] proved that for Fatou's function $f(z):=z+1+e^{-z}$ the escaping set is a spider's web but the fast escaping set is not, hence providing a simple example of a function having this property. This result was subsequently generalised in [ES19] to a larger class of functions.

Our goal in this paper is to transfer this study to the class of transcendental self-maps of $\mathbb{C}^{*}$. It can be shown that such a function $f$ can be written in the form

$$
f(z)=z^{n} \exp (g(z)+h(1 / z))
$$

with $n \in \mathbb{Z}$ and $g, h$ non-constant entire functions; see Råd53, p.88]. Every holomorphic self-map $f$ of $\mathbb{C}^{*}$ can be semiconjugated to an entire function $\tilde{f}$ by the exponential map, that is, the following diagram commutes:

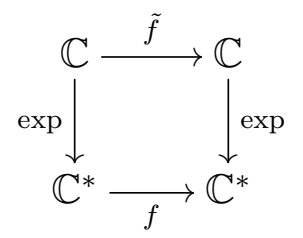

The function $\tilde{f}$ is called a lift of $f$.

We propose the following definition of a spider's web in the punctured plane. We say that a set $X \subset \mathbb{C}^{*}$ is bounded in $\mathbb{C}^{*}$ if its closure in $\widehat{\mathbb{C}}$ does not meet $\{0, \infty\}$; otherwise we say that $X$ is unbounded in $\mathbb{C}^{*}$.

Definition 1.2 ( $\mathbb{C}^{*}$-spider's web). We say that a set $E \subset \mathbb{C}^{*}$ is a $\mathbb{C}^{*}$-spider's web if $E$ is connected, and there exists a sequence of domains $\left(G_{n}^{\prime}\right)_{n \in \mathbb{N}}$, each of which is bounded in $\mathbb{C}^{*}$, such that

(a) for each $n \in \mathbb{N}$, the set $G_{n}^{\prime}$ is doubly connected and separates zero from infinity;

(b) $G_{n}^{\prime} \subset G_{n+1}^{\prime}$ and $\partial G_{n}^{\prime} \subset E$, for $n \in \mathbb{N}$;

(c) $\bigcup_{n \in \mathbb{N}} G_{n}^{\prime}=\mathbb{C}^{*}$.

Our first two results indicate that this definition of a $\mathbb{C}^{*}$-spider's web is correct. The first shows that $\mathbb{C}^{*}$-spiders' webs lift in an obvious sense; see (2).

Theorem 1.3. If $E$ is a spider's web, then $E^{\prime}:=\exp (E)$ is a $\mathbb{C}^{*}$-spider's web. Similarly, if $E^{\prime}$ is a $\mathbb{C}^{*}$-spider's web, then $E:=\exp ^{-1}\left(E^{\prime}\right)$ is a spider's web.

The second relates to a recent result of Evdoridou and Rempe-Gillen ER18, Theorem 1.5]. They showed that a connected set is a spider's web if and only if it separates each point of $\mathbb{C}$ from $\{\infty\}$. Here if $E, X, Y \subset \widehat{\mathbb{C}}$, then we say that $E$ separates $Y$ from $X$ if there is an open set $U$ containing $Y$, such that the closure of $U$ in $\widehat{\mathbb{C}}$ does not meet $X$, and such that $\partial U \subset E$. If $Y$ is the singleton $\{z\}$, then we omit the braces and say that $E$ separates $z$ from $X$. (Note that while this is not the standard topological definition, it can be shown that this is equivalent.) Our second result shows that an equivalent result to [ER18, Theorem 1.5] holds for $\mathbb{C}^{*}$-spiders' webs. 
Theorem 1.4. Let $E \subset \mathbb{C}^{*}$ be connected. Then $E$ is a $\mathbb{C}^{*}$-spider's web if and only if it separates each point of $\mathbb{C}^{*}$ from $\{0, \infty\}$.

The concept of a $\mathbb{C}^{*}$-spider's web is useful if there are dynamically defined and interesting sets that have this structure. In fact, there are many holomorphic selfmaps of $\mathbb{C}^{*}$ for which is a $\mathbb{C}^{*}$-spider's web. To see this, let $\tilde{f}$ be a lift of a holomorphic self-map $f$ of $\mathbb{C}^{*}$. Although it had been discussed earlier, Bergweiler [Ber95] was the first to prove rigorously that $J(f)=\exp J(\tilde{f})$. It follows from Theorem 1.3 that $J(f)$ is a $\mathbb{C}^{*}$-spider's web exactly when $J(\tilde{f})$ is a spider's web. To give a particular example, Osborne Osb13 showed that $J(\tilde{f})$ is a spider's web when $\tilde{f}(z)=\sin z$. Therefore, we can deduce that $J(f)$ is a $\mathbb{C}^{*}$-spider's web for the function

$$
f(z):=\exp \left(\frac{1}{2}\left(z-\frac{1}{z}\right)\right) .
$$

It follows from the above that, in a sense, there seems little further to say about transcendental self-maps of $\mathbb{C}^{*}$ whose Julia set is a $\mathbb{C}^{*}$-spider's web. Accordingly, we now focus on the question of whether the escaping set of a transcendental self-map of $\mathbb{C}^{*}$ can be a $\mathbb{C}^{*}$-spider's web. First we need to define this set. The escaping set of a transcendental self-map $f$ of $\mathbb{C}^{*}$ is given by

$$
I(f):=\left\{z \in \mathbb{C}^{*}: \omega(z, f) \subset\{0, \infty\}\right\},
$$

where $\omega(z, f):=\bigcap_{n \in \mathbb{N}} \overline{\left\{f^{k}(z): k \geq n\right\}}$, and this closure is taken in $\widehat{\mathbb{C}}$. See Mar18, FM17, Mar19, EMS19 for several properties about this set. Observe that, in general, we cannot assume that $I(f)=\exp I(\tilde{f})$ whenever $\tilde{f}$ is a lift of a holomorphic selfmap $f$ of $\mathbb{C}^{*}$; see (2). A counter-example is given when $f(z)=z e^{z-1}$ and $\tilde{f}(z)=$ $z+e^{z}-1+2 \pi i$. Then $\tilde{f}^{n}(0)=2 \pi i n$, for $n \in \mathbb{N}$, and so $0 \in I(\tilde{f})$. However $1=e^{0}$ is a fixed point of $f$. Forthcoming work by Martí-Pete [Mar will consider similar examples.

We then have the following, which is the main result of this paper. This result is based on a function first considered in [Mar19, Example 3.3] which has a hyperbolic Baker domain containing a right half-plane; we leave the definition of these terms for later. See Figure 1 .

Theorem 1.5. There exists $\lambda_{0}>0$ such that if $\lambda \geq \lambda_{0}$, then the transcendental self-map of $\mathbb{C}^{*}$ given by

$$
f_{\lambda}(z):=\lambda z \exp \left(e^{-z} / z\right)=\lambda z \exp \left(\frac{e^{-z}-1}{z}+\frac{1}{z}\right),
$$

has the property that $I\left(f_{\lambda}\right)$ is a $\mathbb{C}^{*}$-spider's web.

Martí-Pete also defined the fast escaping set $A(f)$ for a transcendental self-map $f$ of $\mathbb{C}^{*}$; see Mar18, Definition 1.2]. The definition is, of necessity, quite complicated and so we omit it here. (Note that in this paper we only use the fact that the set $A(f)$ is completely invariant under $f$.) Roughly speaking, $A(f)$ contains those points which tend to $\{0, \infty\}$ eventually faster than a combination of iterates of either the maximum or the minimum modulus functions. It is then quite straightforward to 

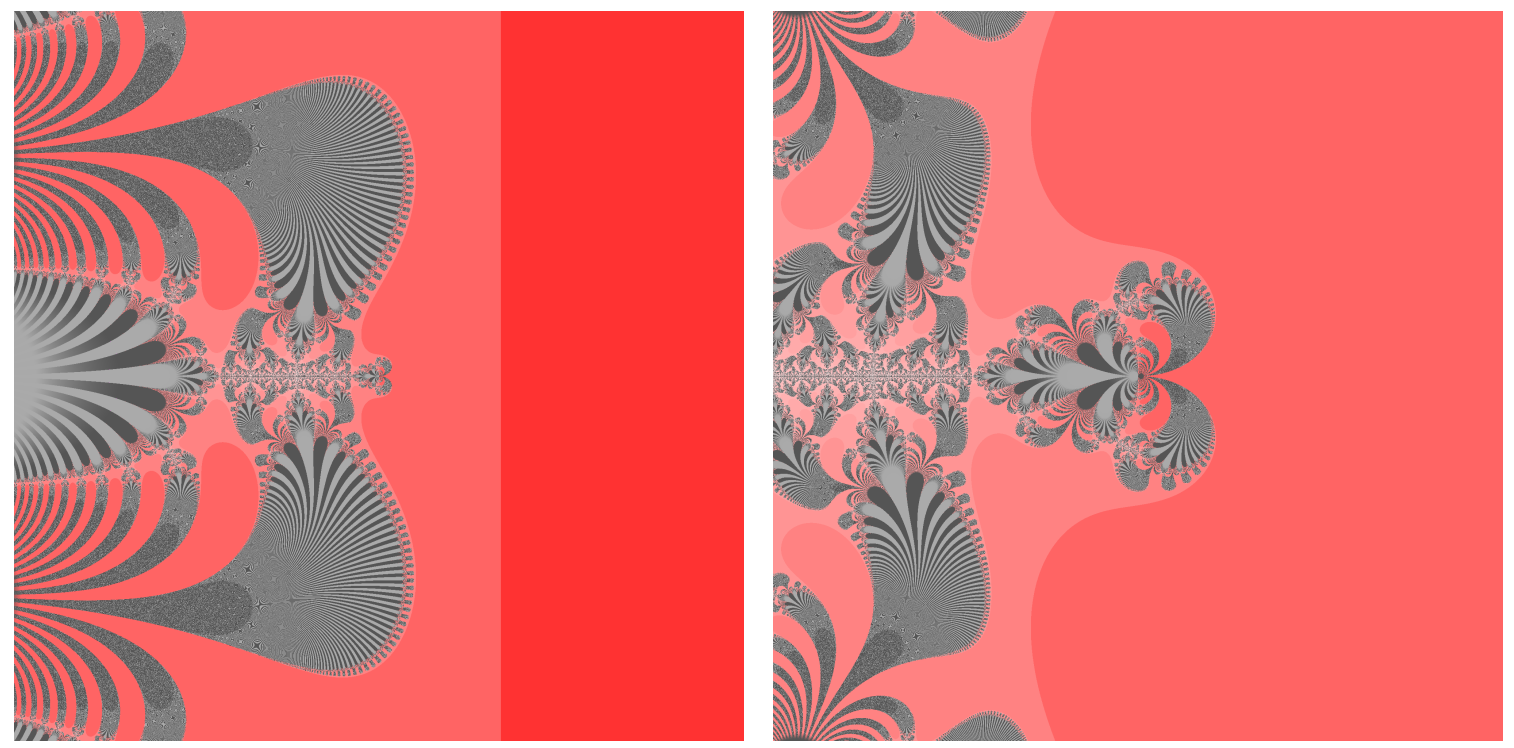

Figure 1. Dynamical plane of the function $f_{\lambda}$ for $\lambda=32$. The Julia set is drawn in gray, and the Baker domain is coloured red. The different shades of red indicate the smallest $n$ for which $\operatorname{Re} f_{\lambda}^{n}(z) \geqslant 2$. On the left, $z \in[-6,6]+i[-6,6]$, and on the right, $z \in[-1,1]+i[-1,1]$.

see that if $f$ is the function in Theorem 1.5 , then $A(f)$ is not a $\mathbb{C}^{*}$-spider's web; this is because there is a right half-plane in which $f$ behaves like $z \mapsto \lambda z$ and so none of these points can escape faster than the maximum modulus. Thus, this function provides a positive answer to the analogue of Question 2 in our setting.

It is also possible to define an analogue of the level set $A_{R}(f)$, though this is complicated and we refer again to [Mar18, Definition 1.2]. However, it is not difficult to show that if $f$ is a transcendental self-map of $\mathbb{C}^{*}$, then $A_{R}(f)$ cannot be a $\mathbb{C}^{*}$ spider's web. Very roughly, by way of contradiction, we let $\Gamma$ be a "loop" in $A_{R}(f)$ which surrounds and is very close to the origin. Then $f(\Gamma)$ must contain a point of very large modulus and also a point of very small modulus. Hence $f(\Gamma)$ contains a point of modulus 1 , and so the preimage of this point on $\Gamma$ cannot lie in $A_{R}(f)$. This gives the necessary contradiction.

Recall that all known examples of transcendental entire functions $f$ for which $A(f)$ is a spider's web have been constructed by first showing that $A_{R}(f)$ is a spider's web. From the previous paragraph, it is clear that an analogous construction is not possible in the case of a transcendental self-map of $\mathbb{C}^{*}$. In view of this fact, together with Question 1, we make the following conjecture.

Conjecture 1. If $f$ is a transcendental self-map of $\mathbb{C}^{*}$, then $A(f)$ is not a $\mathbb{C}^{*}$-spider's web.

Note that our definition of a $\mathbb{C}^{*}$-spider's web is topological. In Six18, Theorem 1.5] it was shown that for many sets it is possible to give a dynamical definition of a spider's web; for example, if $f$ is a transcendental entire function, then $I(f)$ is a spider's web if and only if it separates some point of $J(f)$ from infinity. Our final 
result takes [Six18, Theorem 1.5] into the $\mathbb{C}^{*}$ setting and will be the main tool to prove Theorem 1.5 .

Theorem 1.6. Suppose that $f$ is a transcendental self-map of $\mathbb{C}^{*}$. Then $I(f)$ is a $\mathbb{C}^{*}$-spider's web if and only if it separates some point of $J(f)$ from $\{0, \infty\}$. This statement is also true if we replace $I(f)$ with either $A(f)$ or $J(f)$.

Remark 1.7. Note that in the case of $J(g)$, where $g$ is a transcendental entire function, Six18, Theorem 1.5] requires the additional hypothesis that $g$ has no multiply connected Fatou components. Although a holomorphic self-map $f$ of $\mathbb{C}^{*}$ can have (at most one) multiply connected Fatou component, we do not require this additional hypothesis. In fact, it follows from Propositions 4.2 and 4.4 that if $f$ has a multiply connected Fatou component, then $J(f)$ separates no point of $J(f)$ from $\{0, \infty\}$.

Structure. In Section 2 we give the proof of Theorem 1.4. We then use this result in Section 3 to prove Theorem 1.3. We prove Theorem 1.6 in Section 4, and we use this result to prove Theorem 1.5 in Section 5 .

Notation. Unless otherwise stated, all topological operations such as closure and boundary are taken in $\mathbb{C}$. Also, if $S \subset \mathbb{C}^{*}$, then we denote by $T(S)$ the set formed by appending to $S$ all components of $\widehat{\mathbb{C}} \backslash S$ that do not contain either 0 or $\infty$.

Acknowledgments. We are grateful to the referees for their detailed reading and much helpful feedback; in particular, for comments leading to Remark 4.3 .

\section{Proof of Theorem 1.4}

In this section we prove Theorem 1.4. In fact we prove a slightly more detailed result, which is analogous to [ER18, Theorem 2.10]. Note that in this result we do not assume, initially, that $E$ is connected.

Theorem 2.1. Suppose that $E \subset \mathbb{C}^{*}$. Then the following are equivalent.

(a) There is a sequence of domains $\left(G_{n}^{\prime}\right)_{n \in \mathbb{N}}$, as in the definition of a $\mathbb{C}^{*}$-spider's web.

(b) E separates every compact set in $\mathbb{C}^{*}$ from $\{0, \infty\}$.

(c) E separates every point $z \in \mathbb{C}^{*}$ from $\{0, \infty\}$.

Suppose finally that one of these equivalent conditions holds. Then $E$ is connected (that is, $E$ is a $\mathbb{C}^{*}$-spider's web) if and only if $E \cup\{0, \infty\}$ is connected.

Proof. It is immediate that (b) implies (c). To see that (a) implies (b), let $\left(G_{n}^{\prime}\right)_{n \in \mathbb{N}}$ be the domains from the definition of a $\mathbb{C}^{*}$-spider's web. Suppose that $X \subset \mathbb{C}^{*}$ is compact; in particular $X$ is bounded away from 0 and $\infty$, and so we can choose $n \in \mathbb{N}$, such that $X \subset G_{n}^{\prime}$. Then $G_{n}^{\prime}$ is the necessary open set in the definition of a separation.

Next suppose that (b) holds. We claim that if $K \subset \mathbb{C}^{*}$ is compact, and 0 and $\infty$ lie in different components of $\widehat{\mathbb{C}} \backslash K$, then there is a domain $G=G(K)$ that is bounded in $\mathbb{C}^{*}$, with $K \subset G$ and $\partial G \subset E$, and such that $\widehat{\mathbb{C}} \backslash G$ has exactly two components, 
one containing 0 and one containing $\infty$. To see this we first, by assumption and by definition, let $U$ be an open set, bounded in $\mathbb{C}^{*}$, such that $K \subset U$ and $\partial U \subset E$. We then set $G=T(U)$.

So we can define a sequence $\left(G_{n}^{\prime}\right)_{n \in \mathbb{N}}$ of domains (from the definition of a $\mathbb{C}^{*}$ spider's web) inductively as follows. First we let $K_{1}$ be the circle $\left\{z \in \mathbb{C}^{*}:|z|=1\right\}$. Now suppose that $n \in \mathbb{N}$ and that $K_{n}$ has been defined. We set $G_{n}^{\prime}=G\left(K_{n}\right)$, and we set

$$
K_{n+1}:=T\left(\{z \in \mathbb{C}: 1 / n \leq|z| \leq n\} \cup \overline{G_{n}^{\prime}}\right) .
$$

The domains $G_{n}^{\prime}, n \in \mathbb{N}$, satisfy the requirements in the definition of a $\mathbb{C}^{*}$-spider's web, so (a) holds.

The remainder of the proof is very similar to that in [ER18]. Suppose that (c) holds. Let $K \subset \mathbb{C}^{*}$ be a compact set. Then for every $x \in K$ there is an open set $U(x) \subset \mathbb{C}$ that is bounded in $\mathbb{C}^{*}$, such that $x \in U$ and $\partial U(x) \subset E$.

Since $K$ is compact, there exist $k \in \mathbb{N}$ and points $x_{1}, \ldots, x_{k} \in K$ such that

$$
K \subset U:=\bigcup_{j=1}^{k} U\left(x_{j}\right) .
$$

Clearly

$$
\partial U \subset \bigcup_{j=1}^{k} \partial U\left(x_{j}\right) \subset E,
$$

and $U$ is bounded in $\mathbb{C}^{*}$. So $\partial U$ separates $K$ from $\{0, \infty\}$. This completes the proof of the equivalence of the three conditions (a) to (c).

For the final statement, suppose that (a) holds. In one direction, suppose that $E$ is connected. Since $E$ contains points of arbitrarily large and arbitrarily small modulus, we see that $\{0, \infty\}$ lies in the closure of $E$ in $\widehat{\mathbb{C}}$. Hence $E \cup\{0, \infty\}$ is connected. In the other direction, suppose that $E$ is not connected. Then there are disjoint open sets $U, V \subset \mathbb{C}^{*}$ such that $E \subset U \cup V$ and $U, V$ each meet $E$. Without loss of generality, it follows that for all sufficiently large $n \in \mathbb{N}$, the inner boundary component of $G_{n}^{\prime}$ lies in $V$ and the outer boundary component of $G_{n}^{\prime}$ lies in $U$. (Here inner and outer boundary components are understood in the obvious sense, and recall that, by definition, $G_{n}^{\prime}$ is doubly connected.) Let $V^{\prime}$ denote the union of $V$ with the component of $\widehat{\mathbb{C}} \backslash V$ containing 0 , and let $U^{\prime}$ denote the union of $U$ with the component of $\widehat{\mathbb{C}} \backslash U$ containing $\infty$. Then the open sets $U^{\prime}, V^{\prime}$ disconnect the set $E \cup\{0, \infty\}$, as required.

\section{Proof of Theorem 1.3}

Suppose first that $E$ is a spider's web, and that $\left(G_{n}\right)_{n \in \mathbb{N}}$ are the simply connected domains in the definition of a spider's web, which fill the plane and the boundary of each of which is in $E$. Since $E$ is connected, so is $E^{\prime}=\exp (E)$. To show that $E^{\prime}$ is a $\mathbb{C}^{*}$-spider's web, it remains to construct the domains $\left(G_{n}^{\prime}\right)_{n \in \mathbb{N}}$ in the definition. 
For $R>0$, let $S_{R}$ denote the closed square whose centre is the origin

$$
S_{R}:=\{z \in \mathbb{C}: \max \{|\operatorname{Re} z|,|\operatorname{Im} z|\} \leq R\} .
$$

Choose $R_{1}>2 \pi$ and let $n_{1} \in \mathbb{N}$ be sufficiently large that $S_{R_{1}} \subset G_{n_{1}}$. Then we set

$$
G_{1}^{\prime}:=T\left(\exp \left(G_{n_{1}}\right)\right) \supset \exp \left(S_{R_{1}}\right)=\left\{z \in \mathbb{C}^{*}: e^{-R_{1}} \leq|z| \leq e^{R_{1}}\right\} .
$$

Now, inductively suppose that $k \in \mathbb{N}$, and that $R_{k}, n_{k}$, and $G_{k}^{\prime}$ have all been defined. Choose $R_{k+1}>R_{k}$ sufficiently large that $G_{k}^{\prime} \subset \exp \left(S_{R_{k+1}}\right)$. Let $n_{k+1} \in \mathbb{N}$ be sufficiently large that $S_{R_{k+1}} \subset G_{n_{k+1}}$. Then we set

$$
G_{k+1}^{\prime}:=T\left(\exp \left(G_{n_{k+1}}\right)\right) \supset \exp \left(S_{R_{k+1}}\right)=\left\{z \in \mathbb{C}^{*}: e^{-R_{k+1}} \leq|z| \leq e^{R_{k+1}}\right\} .
$$

It is then straightforward to check that the sequence $\left(G_{n}^{\prime}\right)_{n \in \mathbb{N}}$ has the necessary properties, and this completes the proof in one direction.

In the other direction, suppose that $E^{\prime}$ is a $\mathbb{C}^{*}$-spider's web, and that $\left(G_{n}^{\prime}\right)_{n \in \mathbb{N}}$ are the domains in the definition of a $\mathbb{C}^{*}$-spider's web. Let $E:=\exp ^{-1}\left(E^{\prime}\right)$. We need to show first that $E$ is connected. Without loss of generality, we can assume that $G_{n-1}^{\prime}$ is compactly contained in $G_{n}^{\prime}$, for $n \geq 2$. For $n \in \mathbb{N}$, the set $G_{n}:=\exp ^{-1}\left(G_{n}^{\prime}\right)$ is a $2 \pi i$-periodic "vertical strip" the boundary of which lies in $E$. We claim first that $H:=\bigcup_{n \in \mathbb{N}} \partial G_{n} \subset E$ lies in one component of $E$. For, suppose not. It follows that there exists $n \in \mathbb{N}$ such that the two boundary components of $G_{n}$ lie in different components of $E$. In other words, there is an open set $U$, the boundary of which does not meet $E$, and that contains exactly one component of $\partial G_{n}$. Then $\exp (U)$ separates $E^{\prime}$, which is a contradiction.

Now suppose that $E$ is not connected. Let $T$ be a component of $E$ that does not meet $H$. Then there is an open set $U$, the boundary of which does not meet $E$, that contains $T$ and that lies in $G_{n}$, for some $n \in \mathbb{N}$. Then $\exp (U)$ lies in $G_{n}^{\prime}$ and separates $E^{\prime}$, which is again a contradiction. Thus $E$ is indeed connected.

Now suppose that $\zeta \in \mathbb{C}$. Since $E^{\prime}$ is a $\mathbb{C}^{*}$-spider's web it follows from Theorem 2.1 that there is an open set $U^{\prime}$, bounded in $\mathbb{C}^{*}$, that contains $\exp (\zeta)$ and the boundary

of which lies in $E^{\prime}$. Let $U$ be the component of $\exp ^{-1}\left(U^{\prime}\right)$ containing $\zeta$. Then $U$ is a bounded open set, containing $\zeta$, the boundary of which lies in $E$. It follows by [ER18, Theorem 2.10] that $E$ is a spider's web.

\section{Proof of Theorem 1.6}

In this section we require the following. This is the $\mathbb{C}^{*}$ analogue of the blowing-up property, which is well-known for rational and transcendental entire functions. In $\mathbb{C}^{*}$, this result is due to Rådström Råd53, Theorem 4.1]. Note that a transcendental self-map of $\mathbb{C}^{*}$ has no Picard exceptional values.

Lemma 4.1. Suppose that $f$ is a transcendental self-map of $\mathbb{C}^{*}$. Suppose also that $U \subset \mathbb{C}^{*}$ is an open set which meets $J(f)$, and that $K \subset \mathbb{C}^{*}$ is a compact set. Then there exists $n_{0}=n_{0}(K, U) \in \mathbb{N}$ such that $f^{n}(U) \supset K$ for all $n \geq n_{0}$.

First we prove the following, which is quite general. Here if $f: X \rightarrow X$ is a function, a set $Y \subset X$ is forward invariant if $f(Y) \subset Y$. 
Proposition 4.2. Suppose that $f$ is a transcendental self-map of $\mathbb{C}^{*}$, and that the set $X \subset \mathbb{C}^{*}$ is forward invariant. Then $X$ contains $a \mathbb{C}^{*}$-spider's web if and only if it separates some point of $J(f)$ from $\{0, \infty\}$.

Proof. One direction is immediate; it follows from the definitions that a set separates every point of $\mathbb{C}^{*}$ from $\{0, \infty\}$ if it contains a $\mathbb{C}^{*}$-spider's web.

In the other direction, suppose that $X$ separates a point of $J(f)$ from $\{0, \infty\}$. In other words, there is an open set $U$, bounded in $\mathbb{C}^{*}$, that meets $J(f)$, and the boundary of which lies in $X$. We now inductively construct a sequence $\left(S_{n}\right)_{n \in \mathbb{N}}$ of subsets of $X$, each of which is bounded in $\mathbb{C}^{*}$.

For the first step in the induction, by Lemma 4.1, there exists $p_{1} \in \mathbb{N}$ such that

$$
\left\{z \in \mathbb{C}^{*}: 1 / 2 \leq|z| \leq 2\right\} \subset f^{p_{1}}(U) .
$$

We set $S_{1}:=\partial T\left(f^{p_{1}}(U)\right) \subset \partial f^{p_{1}}(U) \subset f^{p_{1}}(\partial U) \subset f^{p_{1}}(X) \subset X$.

Now suppose that $S_{n}$ has been defined for some $n \in \mathbb{N}$. Choose $N=N(n) \in \mathbb{N}$ sufficiently large that

$$
S_{n} \subset\left\{z \in \mathbb{C}^{*}: 1 / 2^{N} \leq|z| \leq 2^{N}\right\}
$$

Next, by Lemma 4.1 again, there exists $p_{n+1} \in \mathbb{N}$ such that

$$
\left\{z \in \mathbb{C}^{*}: 1 / 2^{N} \leq|z| \leq 2^{N}\right\} \subset f^{p_{n+1}}(U) .
$$

We set $S_{n+1}:=\partial T\left(f^{p_{n+1}}(U)\right) \subset X$.

Now, consider the image $f\left(S_{n}\right)$, for $n \in \mathbb{N}$. Observe that $S_{n}$ has two components, and if $n$ is sufficiently large one component contains only points of small modulus, and the other component contains only points of large modulus. Hence, if $n$ is sufficiently large, the image of each of these components contains a point of very small modulus and another point of very large modulus. Thus there exists $n_{0} \in \mathbb{N}$ such that the set

$$
S:=\bigcup_{n=n_{0}}^{\infty}\left(S_{n} \cup f\left(S_{n}\right)\right),
$$

is connected. Note that since $X$ is forward invariant we have that $S \subset X$.

We claim that $S$ is a $\mathbb{C}^{*}$-spider's web. To see this, in the definition of a $\mathbb{C}^{*}$-spider's web we let $G_{n}^{\prime}$ be the component of $\widehat{\mathbb{C}} \backslash S_{n}$ that is bounded in $\mathbb{C}^{*}$. It is easy to see that these sets have the necessary properties.

Remark 4.3. Note that Proposition 4.2 does not have an analogue for transcendental entire functions in general. To see this, suppose that $f$ is a transcendental entire function with a multiply connected Fatou component. It is well-known, see, for example, [Bak84], that there is a Jordan curve $\gamma \subset F(f) \cap I(f)$ such that $f^{n}(\gamma)$ surrounds $f^{n-1}(\gamma)$, for $n \in \mathbb{N}$. Then $X:=\bigcup_{n=0}^{\infty} f^{n}(\gamma)$ is a forward invariant set that separates every point of $J(f)$ from infinity. However, $X$ does not contain a spider's web. 
We now show that, similarly as for a transcendental entire function, if the escaping set contains a $\mathbb{C}^{*}$-spider's web, then it is a $\mathbb{C}^{*}$-spider's web.

Proposition 4.4. Suppose that $f$ is a transcendental self-map of $\mathbb{C}^{*}$. If $I(f)$ contains $a \mathbb{C}^{*}$-spider's web, then $I(f)$ is a $\mathbb{C}^{*}$-spider's web. This statement is also true if we replace $I(f)$ with either $A(f)$ or $J(f)$.

Proof. The cases of $A(f)$ and $J(f)$ are immediate, since each component of these sets is unbounded in $\mathbb{C}^{*}$; see [Mar18, Theorem 1.5] and [BD98, Theorem 2], respectively.

The technique for $I(f)$ is inspired by the remark [RS12, p.807]. Suppose that $I(f)$ contains a $\mathbb{C}^{*}$-spider's web, say $E$. We can assume that $E$ is a component of $I(f)$. Since all components of $A(f)$ are unbounded in $\mathbb{C}^{*}$, we see that each component of $A(f)$ meets $E$. It follows that $A(f) \subset E$, since $E$ is a component of $I(f)$. We show that, in fact, $I(f)=E$, which completes the proof.

Suppose that $z \in I(f) \cap J(f)$. Since $J(f)=\partial A(f)$ [Mar18, Theorem 1.3], it follows that

$$
z \in \partial A(f) \subset \bar{E}
$$

Hence $E \cup\{z\}$ is connected, and so, since $E$ is a component of $I(f)$, we can deduce that $I(f) \cap J(f) \subset E$.

It remains to show that $I(f) \cap F(f) \subset E$. First we show that if $V$ is a Fatou component that meets $I(f)$, then $\partial V$ meets $I(f)$. Clearly if $\partial V \subset I(f)$, then there is nothing to prove. Otherwise there is a point $z \in \partial V$ that is not in $I(f)$. For $n \in \mathbb{N} \cup\{0\}$, let $V_{n}$ be the Fatou component containing $f^{n}(V)$. Let $\left(G_{n}^{\prime}\right)_{n \in \mathbb{N}}$ be the sequence of domains in the definition of a $\mathbb{C}^{*}$-spider's web. It follows that there exist $n_{0}, n \in \mathbb{N}$ such that $f^{n}(z) \in G_{n_{0}}^{\prime} \cap \partial V_{n}$ and $f^{n}(V) \cap\left(\mathbb{C}^{*} \backslash G_{n_{0}}^{\prime}\right) \neq \emptyset$. Note that every neighbourhood of either 0 or $\infty$ contains points of $J(f)$, so $V_{n}$ cannot be a punctured neighbourhood of either of these points. We can deduce that $\partial V_{n}$ meets $\partial G_{n_{0}}^{\prime} \subset E$. Thus $\partial V$ meets $I(f)$, as required.

Let $V$ be a Fatou component in $I(f)$, so that $\partial V$ meets $I(f)$. As $\partial V \subset J(f)$, we know that $\partial V \cap E \neq \emptyset$. The result is now immediate.

Recall that $I(f), A(f)$ and $J(f)$ are all completely invariant. It follows, then, that Theorem 1.6, is an immediate consequence of Proposition 4.2 and Proposition 4.4 .

\section{Proof of Theorem 1.5}

We use the following result Mar18, Lemma 4.1], which is a version of RS11, Lemma 1].

Lemma 5.1. Suppose that $\left(E_{n}\right)_{n \geq 0}$ is a sequence of compact subsets of $\mathbb{C}^{*}$, and that $f: \mathbb{C}^{*} \rightarrow \mathbb{C}^{*}$ is a continuous function such that $f\left(E_{n}\right) \supset E_{n+1}$, for $n \geq 0$. Then there exists $z \in E_{0}$ such that $f^{n}(z) \in E_{n}$, for $n \in \mathbb{N}$.

Recall that we need to prove that if $\lambda>0$ is sufficiently large, then $I\left(f_{\lambda}\right)$ is a $\mathbb{C}^{*}$-spider's web for the transcendental self-map of $\mathbb{C}^{*}$ given by

$$
f_{\lambda}(z):=\lambda z \exp \left(e^{-z} / z\right)
$$


First we note the following. Suppose that $\lambda>0$, and $z=x+i y$. Then

$$
\frac{e^{-z}}{z}=\frac{e^{-x}}{x^{2}+y^{2}}((x \cos y-y \sin y)-i(x \sin y+y \cos y))=: \xi+\zeta i .
$$

We obtain that

$$
\begin{aligned}
& \operatorname{Re} f_{\lambda}(z)=\lambda e^{\xi}(x \cos \zeta-y \sin \zeta), \\
& \operatorname{Im} f_{\lambda}(z)=\lambda e^{\xi}(x \sin \zeta+y \cos \zeta),
\end{aligned}
$$

and

$$
\left|f_{\lambda}(z)\right|=\lambda|z| \exp \left(\frac{e^{-x}}{x^{2}+y^{2}}(x \cos y-y \sin y)\right) .
$$

We frequently use the fact, without comment, that the function $f_{\lambda}$ is symmetric with respect to the real line, that is, $f_{\lambda}(\bar{z})=\overline{f_{\lambda}(z)}$ for $z \in \mathbb{C}^{*}$.

We say that a Fatou component $U$ is a Baker domain if $U \subseteq I(f)$ and $U$ is periodic, that is, $f^{p}(U) \subseteq U$ for some $p \in \mathbb{N}$. In the case that $p=1$, we say that $U$ is an invariant Baker domain. In [Mar19, Example 3.3] it was shown that for $\lambda \geq 2$, the function $f_{\lambda}$ has a hyperbolic invariant Baker domain containing a right half-plane. Roughly speaking, this means that $f_{\lambda}$ behaves like $z \mapsto \lambda z$ on a right half-plane; we refer to the survey |Rip08] for a more detailed classification of Baker domains, and see also [Kön99, Ber01, FH06]. For the sake of completeness, we include a proof that $f_{\lambda}$ has a Baker domain.

Lemma 5.2. Suppose that $\lambda \geq 2$. Then $\operatorname{Re} f_{\lambda}(z) \geq 0.7 \lambda \operatorname{Re} z$, for $\operatorname{Re} z \geq 2$. In particular $f_{\lambda}$ has a Baker domain containing a right half-plane.

Proof. We have that

$$
f_{\lambda}(z)=\lambda z\left(1+\frac{1}{z e^{z}}+\frac{1}{2 !\left(z e^{z}\right)^{2}}+\frac{1}{3 !\left(z e^{z}\right)^{3}} \cdots\right) .
$$

Suppose that $z=x+i y$ is such that $x \geq 2$. Then $\left|z e^{z}\right| \geq x \cdot e^{x} \geq 2 e^{2} \geq 10$. Also $\left|e^{z}\right| \geq e^{2} \geq 7$. We can assume that $y \geq 0$. Hence

$$
\begin{aligned}
\operatorname{Re} f_{\lambda}(z) & =\lambda x \operatorname{Re}\left(1+\frac{1}{z e^{z}}+\frac{1}{2 !\left(z e^{z}\right)^{2}}+\frac{1}{3 !\left(z e^{z}\right)^{3}} \ldots\right)-\lambda y \operatorname{Im}\left(\frac{\frac{1}{e^{z}}+\frac{1}{2 ! z e^{2 z}}+\frac{1}{3 ! z^{2} e^{3 z}} \cdots}{z}\right) \\
& \geq \lambda x\left(1-\frac{1}{10}-\frac{1}{200}-\ldots\right)-\lambda\left(\frac{1}{7}+\frac{1}{4 \cdot 49}+\ldots\right) \\
& \geq 0.8 \lambda x-0.2 \lambda \\
& \geq 0.7 \lambda x .
\end{aligned}
$$

The claim follows.

From now on, for simplicity, we write $f$ instead of $f_{\lambda}$, and we will assume that $\lambda \geq 2$. Next we define a closed subset $I$ of $I(f)$ and show that all its complementary components are bounded in $\mathbb{C}^{*}$. Note that Theorem 1.5 follows from this fact. To see this, let $z \in \mathbb{C}^{*}$ be a repelling periodic point of $f$ and let $W$ be the component of 
$\mathbb{C}^{*} \backslash I$ that contains $z$. Then $W$ is a domain that contains $z$ and is bounded in $\mathbb{C}^{*}$, with $\partial W \subset I(f)$. Thus $I(f)$ is indeed a $\mathbb{C}^{*}$-spider's web, by Theorem 1.6 .

To define our set $I$, first we set

$$
H:=\left\{z \in \mathbb{C}^{*}: \operatorname{Re} z \geq 2\right\},
$$

which is an absorbing domain. In other words, for each point $z$ in the Baker domain, there exists $n_{0} \in \mathbb{N}$ such that $f^{n}(z) \in H$ for $n \geq n_{0}$. Then, we define

$I:=\left\{z \in \mathbb{C}^{*}:\right.$ for all $n \in \mathbb{N}$, either $\left|f^{n}(z)\right| \geq n / 2$ or $\left|f^{n}(z)\right| \leq 2 / n$ or $\left.f^{n+2}(z) \in H\right\}$.

(Note that in the definition of $I$ we allow the possibility that there are values of $n$ for which two of the conditions hold simultaneously.) Observe that $I$ is forward invariant and contains $f^{-2}(H)$; we will use these properties in the below.

It follows from Lemma 5.2 that $I$ is a closed subset of $I(f)$, and so it remains to show that all components of $\mathbb{C}^{*} \backslash I$ are bounded in $\mathbb{C}^{*}$; see Figure 2 .

The following observations are trivial, and the proofs are omitted.

Observation 5.3. Suppose that $z \in \mathbb{C}^{*} \backslash I$ and $k \in \mathbb{N}$ are such that either $\left|f^{n}(z)\right| \geq n / 2$ or $\left|f^{n}(z)\right| \leq 2 / n$, for $1 \leq n<k$. Then $f^{k}(z) \notin f^{-2}(H)$.

Observation 5.4. We have that $f\left(\mathbb{R}^{+}\right) \subset H$, and so $f^{-1}\left(\mathbb{R}^{+}\right) \subset f^{-2}(H)$.
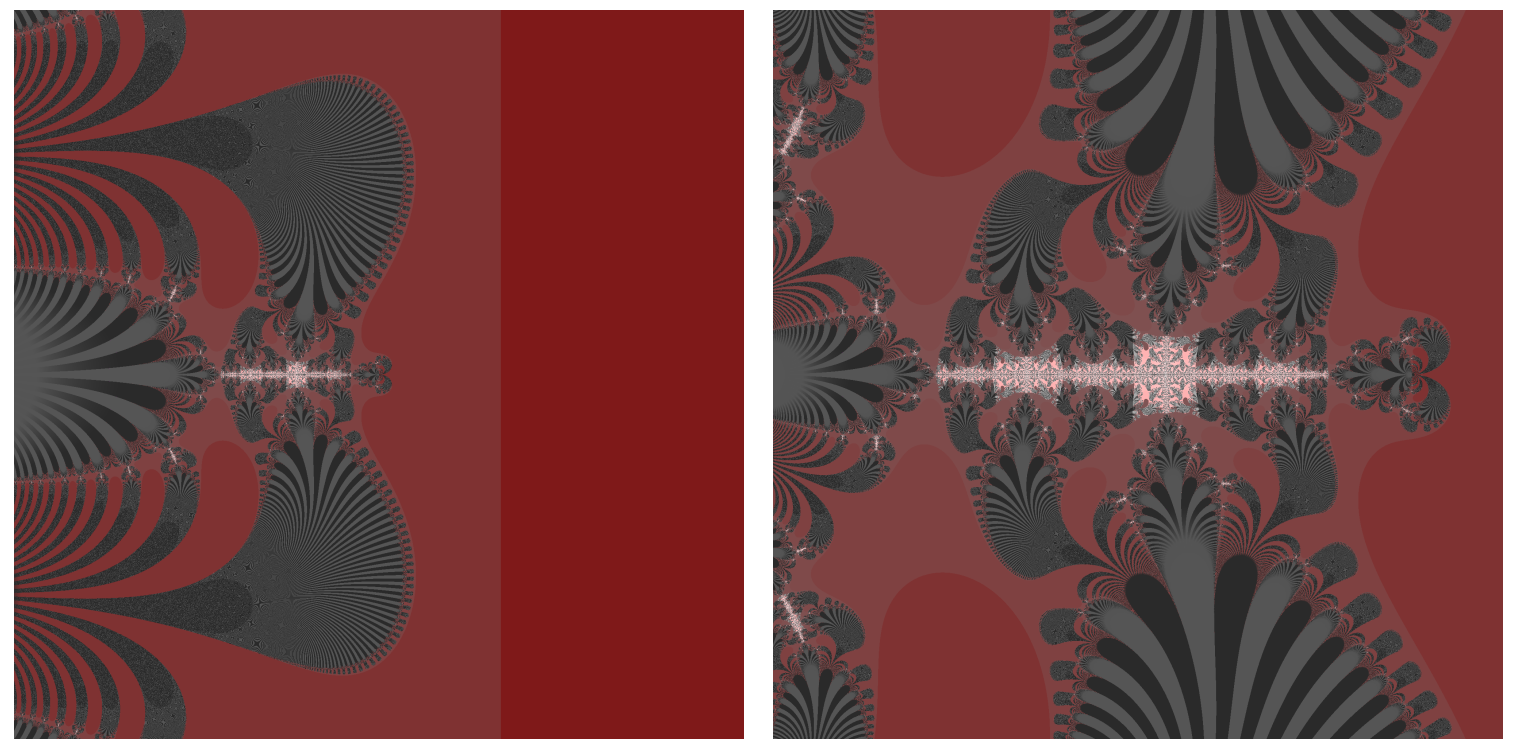

Figure 2. The complement of the closed set $I \subset I\left(f_{\lambda}\right)$ for $\lambda=32$ is depicted in light colours. On the left, $z \in[-6,6]+i[-6,6]$. On the right, a zoom of the largest component containing the two repelling fixed points on the negative real axis; here $z \in[-3.5,0.5]+i[-2,2]$. Note that $I$ is bounded away from zero, though this is hard to show in a figure.

The proof that all components of $\mathbb{C}^{*} \backslash I$ are bounded in $\mathbb{C}^{*}$ is long and complicated, so we begin with a very rough sketch of the strategy. We begin by supposing, by 
way of contradiction, that there is a component, $X$, of $\mathbb{C}^{*} \backslash I$ that is unbounded in $\mathbb{C}^{*}$. Since $X$ is open, this implies that there is a long curve, $\Gamma_{1}$ say, contained in $X$. Note that here, by long we mean that $\Gamma_{1}$ contains points the ratio of whose moduli is large; see Lemma 5.9 for a more precise statement.

By studying the set $f^{-2}(H)$, and by Observation 5.3 , we show that $\Gamma_{1}$ must contain a subcurve, $\Gamma_{1}^{\prime}$, that lies in one of a collection of channels in which either $|f|$ is very large or $|f|$ is very small; these sets are illustrated in Figure 5. We are then able to deduce that $\Gamma_{2}=f\left(\Gamma_{1}^{\prime}\right)$ is a long curve, in the sense mentioned above, which also does not meet $f^{-2}(H)$. Hence we can apply the process above to $\Gamma_{2}$ to obtain another curve $\Gamma_{3}$. We then iterate this process, and hence, using Lemma 5.1, prove the existence of a point $z \in \Gamma_{1} \cap I$, which is a contradiction. This completes the sketch of our proof.

It is clear from the definition of $I$, together with Observation 5.3 , that the preimage $f^{-2}(H)$ plays an important role. Hence, in view of Observation 5.4, we begin by considering the preimage of the positive real axis (see Figure 3 ).

Lemma 5.5. Set $V=f^{-1}\left(\mathbb{R}^{+}\right)$. Then $V$ consists of the following curves, which we call the curves in $V$ :

- The positive real axis $\mathbb{R}^{+}$.

- A collection of curves from 0 to 0 that lie in the unit disc.

- A collection of curves from $\infty$ to $\infty$ that lie outside the unit disc. Moreover, $\operatorname{Re} z \rightarrow-\infty$ and $|\operatorname{Im} z|$ is bounded as $z \rightarrow \infty$ on these curves.

Exactly one of the curves in $V$, apart from $\mathbb{R}^{+}$itself, meets the positive real axis and none meet the negative real axis. Finally, there is a number $t>0$ such that if $z_{1}$ and $z_{2}$ are two points of the same curve in $V$, then $\left|\operatorname{Im} z_{1}-\operatorname{Im} z_{2}\right|<t$.

Remark 5.6. By considering the critical points of $f$, we can in fact show something slightly stronger. Within the unit disc, $V$ contains a curve $\Gamma$ from 0 to 0 that is symmetric in the real axis, which it crosses once near the point 0.8 ; see Figure 3 . All the other curves in $V$ are disjoint, and lie in a left half-plane. However, the proof of these facts is complicated and is omitted, as this extra detail is not required for the proof of our result.

Proof of Lemma 5.5. Suppose that $z=x+i y \in V$, and we can assume that $y \geq 0$. It follows from (4) that

$$
y \cos \left(\frac{e^{-x}}{x^{2}+y^{2}}(x \sin y+y \cos y)\right)=x \sin \left(\frac{e^{-x}}{x^{2}+y^{2}}(x \sin y+y \cos y)\right) .
$$

(Note that the solutions to this equation give preimages of both the positive real line and the negative real line.)

Observe that $V$ is necessarily a union of analytic curves which run from $\{0, \infty\}$ to $\{0, \infty\}$. One of these curves is the positive real axis. We claim that this is the only curve in $V$ that meets the unit circle

$$
\left\{z \in \mathbb{C}^{*}:|z|=1\right\} .
$$



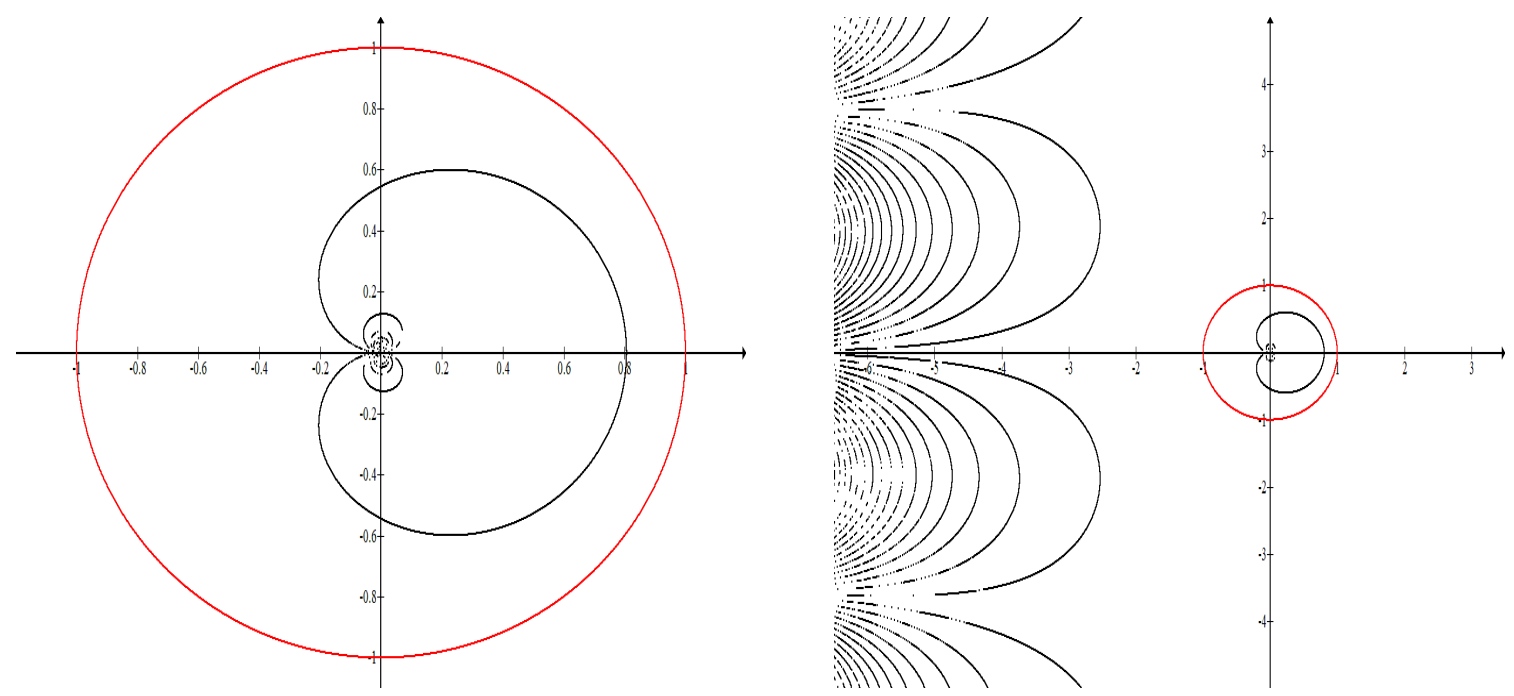

FiguRE 3. Two images of the curves in $V$, showing the unit circle in red.

To prove this, suppose that $x=\cos \theta$ and $y=\sin \theta$ are such that $x+i y$ is in $V$. From (6) we obtain that

$$
\tan \theta=\tan \left(e^{-\cos \theta}(\cos \theta \sin (\sin \theta)+\sin \theta \cos (\sin \theta))\right),
$$

and hence, by the compound angle formula, that

$$
\theta+n \pi=e^{-\cos \theta} \sin (\sin \theta+\theta), \quad \text { for } n \in \mathbb{Z} .
$$

It can then be checked (numerically or by graph drawing) that the only solutions to this equation are when $\theta$ is a multiple of $\pi$, as required. (Note that the point corresponding to $\theta=\pi$ lies on the negative real axis, which is a preimage of the negative real axis.)

It follows that the only curve in $V$ which crosses the unit circle is the positive real axis. Hence all other curve in $V$ run either from 0 to 0 , or from $\infty$ to $\infty$.

To prove the penultimate claim of the lemma, note first that the negative real axis maps under $f$ to the negative real axis. Next we note that the critical points of $f$ occur when

$$
e^{z}=1+\frac{1}{z}
$$

It can then be checked that there is only one critical point on the positive real axis, and this is a simple critical point; this is the point close to 0.8 mentioned in Remark 5.6 and visible in Figure 3. The penultimate claim of the lemma follows.

It remains to consider the behaviour of $V$ near infinity. Observe from (6) that, apart from the positive real line, no curve in $V$ can meet the set of points $z=x+i y$ where

$$
x \sin y+y \cos y=0 .
$$


In polar coordinates this gives

$$
\theta+n \pi=-r \sin \theta, \quad \text { for } n \in \mathbb{Z} .
$$

For large values of $n$, this is approximately the horizontal line

$$
y=r \sin \theta=n \pi, \quad \text { for } n \in \mathbb{Z},
$$

and so no curve in $V$ can tend to infinity by increasing imaginary part. In particular, this gives the final claim of the lemma. It also implies that we can consider separately the solutions to (6) in the two cases that $x$ is large and positive, and $x$ is large and negative.

First, suppose that $x$ is large and positive. Then the terms

$$
\frac{e^{-x} x \sin y}{x^{2}+y^{2}} \text { and } \frac{e^{-x} y \cos y}{x^{2}+y^{2}}
$$

are both small (independently of the size of $y$ ). Thus if (6) holds, then $y$ is small compared to $x$. It follows that, for $x$ large and positive, the solutions to (6) are approximately when

$$
y=e^{-x} \sin y,
$$

from which we can deduce that $y=0$ is the only solution to (6) when $x$ is large and positive. It follows that the curves from $\infty$ to $\infty$ in $V$ must tend to infinity via large, negative real parts, as claimed. This completes the proof.

We will use our next result to prove that certain curves that do not meet $f^{-2}(H)$ must contain points of large (negative) real part.

Lemma 5.7. Suppose that $\lambda \geq 32$. Then there exists $t^{\prime}>0$ with the following property. Suppose that $\Gamma$ is a connected set that does not meet $f^{-2}(H)$, and that $z_{1}, z_{2} \in \Gamma$. Then $\left|\operatorname{Im} z_{1}-\operatorname{Im} z_{2}\right|<t^{\prime}$.

Proof. To prove this result we, in fact, prove the following. We claim that if $\lambda \geq 32$, then for all $n \in \mathbb{Z}$ with $|n|$ sufficiently large, there exists $x_{n}<0$ such that the horizontal half-line

$$
\left\{z=x \pm 2 n \pi i: x \geq x_{n}\right\}
$$

lies in $f^{-2}(H)$ and meets a component of $f^{-1}\left(\mathbb{R}^{+}\right)$; see Figure 4 , which illustrates the case $n=1$. Note that the lemma follows from this claim, by Observation 5.4 and the final part of Lemma 5.5.

To prove this, suppose that $y=2 n \pi$, where $n \in \mathbb{N}$ is large, and $z=x+i y$. Then, by (3),

$$
\operatorname{Re} f(z)=\lambda \exp \left(\frac{e^{-x} x}{x^{2}+y^{2}}\right)\left(x \cos \left(\frac{e^{-x} y}{x^{2}+y^{2}}\right)+y \sin \left(\frac{e^{-x} y}{x^{2}+y^{2}}\right)\right) .
$$




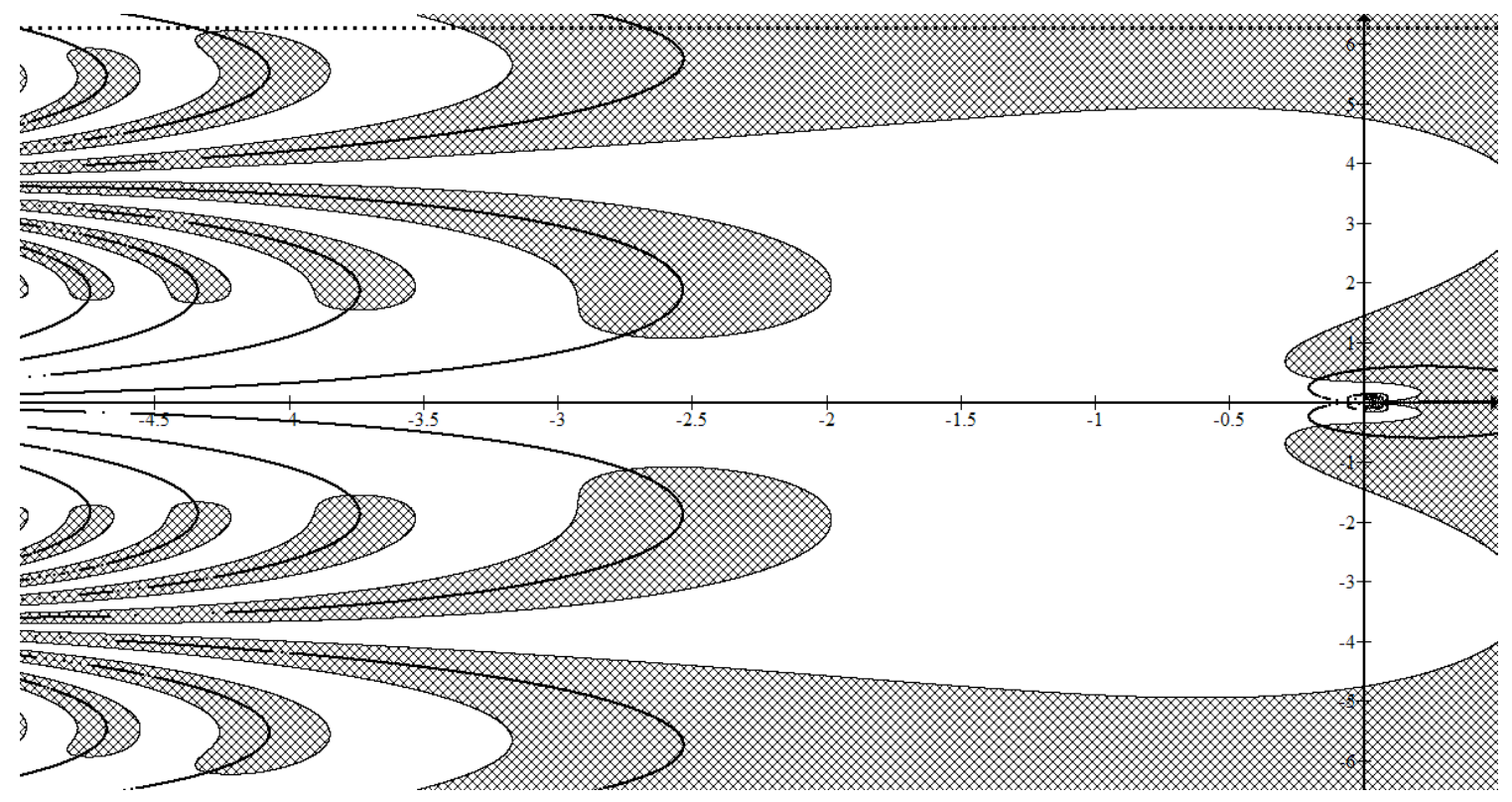

FIGURE 4. Once again, the hatched area is $f_{32}^{-1}(H)$ and the black lines are the preimages of the positive real axis. Note, on the left, the curves that are approximated by the sets $A_{n}^{\prime}$ from Lemma 5.10. Note also that the line $y=2 \pi$, shown dotted, meets a preimage of the positive real axis inside $f_{32}^{-1}(H)$; see Lemma 5.7 .

We now consider three ranges of values of $x$. Recall that $\lambda \geq 32$. Suppose first that $x \geq 1$. If $n>0$ is sufficiently large, then

$$
\operatorname{Re} f(z) \geq \lambda\left(x \cos \left(\frac{e^{-x} y}{x^{2}+y^{2}}\right)+y \sin \left(\frac{e^{-x} y}{x^{2}+y^{2}}\right)\right) \geq \frac{\lambda x}{2} \geq 2 .
$$

Suppose next that $0 \leq x<1$. If $n>0$ is sufficiently large, then

$$
\operatorname{Re} f(z) \geq \lambda\left(x \cos \left(\frac{e^{-x} y}{x^{2}+y^{2}}\right)+y \sin \left(\frac{e^{-x} y}{x^{2}+y^{2}}\right)\right) \geq \frac{\lambda y}{2} \cdot \frac{e^{-x} y}{x^{2}+y^{2}} \geq \frac{\lambda}{4 e} \geq 2
$$

Suppose finally that $-(\log y+\log 2) \leq x<0$. It follows that if $n>0$ is sufficiently large, then, since $|x / y|$ can be assumed to be close to zero, we have

$$
0<\frac{e^{-x} y}{x^{2}+y^{2}}+\arctan (x / y)<2
$$

from which we can deduce that

$$
\sin \left(\frac{e^{-x} y}{x^{2}+y^{2}}+\arctan (x / y)\right)>\frac{e^{-x} y}{4\left(x^{2}+y^{2}\right)} .
$$


It follows that

$$
\begin{aligned}
\operatorname{Re} f(z) & =\lambda \exp \left(\frac{e^{-x} x}{x^{2}+y^{2}}\right)\left(x \cos \left(\frac{e^{-x} y}{x^{2}+y^{2}}\right)+y \sin \left(\frac{e^{-x} y}{x^{2}+y^{2}}\right)\right) \\
& =\lambda \exp \left(\frac{e^{-x} x}{x^{2}+y^{2}}\right) \cdot \sqrt{x^{2}+y^{2}} \cdot \sin \left(\frac{e^{-x} y}{x^{2}+y^{2}}+\arctan (x / y)\right) \\
& \geq \lambda \exp \left(\frac{e^{-x} x}{x^{2}+y^{2}}\right) \cdot \frac{e^{-x} y}{4 \sqrt{x^{2}+y^{2}}} \\
& \geq \lambda \exp \left(\frac{2 x y}{x^{2}+y^{2}}\right) \cdot \frac{y}{4 \sqrt{x^{2}+y^{2}}} \\
& \geq \frac{\lambda}{16} \\
& \geq 2
\end{aligned}
$$

In other words, if $n$ is sufficiently large, then the whole line segment

$$
\{z=x+2 n \pi i:-(\log 2 n \pi+\log 2) \leq x\}
$$

maps into $H$. The proof is complete if we can show that there is a point of $f^{-1}\left(\mathbb{R}^{+}\right)$ somewhere on this line. When $y=2 n \pi$, equation (6) gives

$$
y \cos \left(\frac{e^{-x} y}{x^{2}+y^{2}}\right)=x \sin \left(\frac{e^{-x} y}{x^{2}+y^{2}}\right) .
$$

It follows by a calculation that, when $|n|$ is large, this has a solution close to

$$
x=-(\log y+\log (\pi / 2)) .
$$

This completes the proof.

In the remainder of this paper we shall assume that $\lambda \geq 32$, so that the conclusions of Lemma 5.7 hold.

We now define some sets, which we call channels, where the modulus of $f$ is either very large or very small. Suppose that $R \geq 1$ and, for definiteness, fix $\epsilon_{0}=1 / 4$. We define the following disjoint domains (which depend on $R$ only);

$$
\begin{aligned}
& C^{+}(R):=\left\{z=x+i y \in \mathbb{C}^{*}: x>0,|y|<\epsilon_{0}|x|,|z|<1 / R\right\} \\
& C^{-}(R):=\left\{z=x+i y \in \mathbb{C}^{*}: x<0,|y|<\epsilon_{0}|x|,|z|<1 / R\right\}
\end{aligned}
$$

and for each $n \in \mathbb{Z}$,

$$
C_{n}(R):=\left\{z=x+i y \in \mathbb{C}^{*}: x<-R(|n|+1),|y-n \pi|<\epsilon_{0}\right\} .
$$

Finally we set

$$
C(R):=C^{+}(R) \cup C^{-}(R) \cup \bigcup_{n \in \mathbb{Z}} C_{n}(R) .
$$

See Figure 5 for a rough schematic of these sets. Note that if $1 \leq R<R^{\prime}$, then $C\left(R^{\prime}\right) \subset C(R)$. 

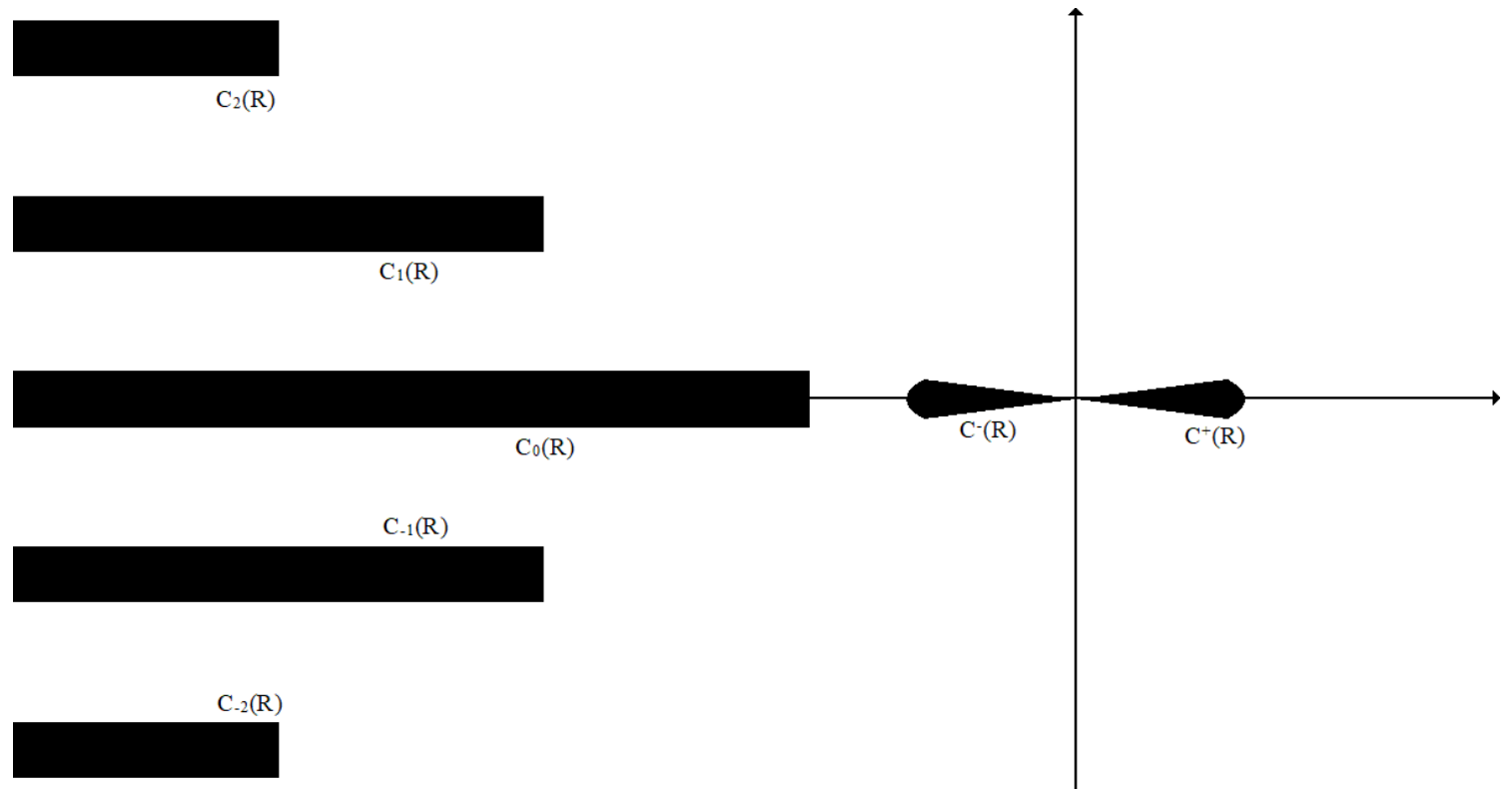

Figure 5. An approximate schematic of the channels that make up $C(R)$.

We then have the following which shows that, in a suitable sense, the function $f$ "blows up" in the channels. Note that in this result a much faster rate of growth is possible, but this is not needed.

Lemma 5.8. There exists $K>1$ such that for all $L>1$, there exist $R_{0}>1$ with the following property. Suppose that $z, z_{0}, z_{1}$ all lie in the same component, $W$ say, of $C\left(R_{0}\right)$. Suppose also that $\left|z_{1} / z_{0}\right| \geq K$. Then:

(1) If $W=C^{+}\left(R_{0}\right)$, then

$$
|f(z)| \geq \frac{L}{|z|} \quad \text { and } \quad\left|\frac{f\left(z_{0}\right)}{f\left(z_{1}\right)}\right| \geq L K .
$$

(2) If $W=C^{-}\left(R_{0}\right)$, then

$$
|f(z)| \leq \frac{|z|}{L} \quad \text { and } \quad\left|\frac{f\left(z_{1}\right)}{f\left(z_{0}\right)}\right| \geq L K .
$$

(3) If $W=C_{2 n}\left(R_{0}\right)$, where $n \in \mathbb{Z}$, then

$$
|f(z)| \geq L|z| \quad \text { and } \quad\left|\frac{f\left(z_{1}\right)}{f\left(z_{0}\right)}\right| \geq L K .
$$

(4) If $W=C_{2 n+1}\left(R_{0}\right)$, where $n \in \mathbb{Z}$, then

$$
|f(z)| \leq \frac{1}{L|z|} \quad \text { and } \quad\left|\frac{f\left(z_{0}\right)}{f\left(z_{1}\right)}\right| \geq L K .
$$

Proof. We begin by fixing values of $t>0$ and $K>1$ sufficiently large that if $z_{0}=x_{0}+i y_{0}$ and $z_{1}=x_{1}+i y_{1}$ both lie in the same component of $C(t)$ and $\left|z_{1} / z_{0}\right| \geq K$, then $\left|x_{1}\right| \geq 8\left|x_{0}\right|$. 
Now suppose that $L>1$. Consider first case (1). Suppose that $z=x+i y$, $z_{0}=x_{0}+i y_{0}$ and $z_{1}=x_{1}+i y_{1}$ are all points of $C^{+}\left(R_{0}\right)$ with $\left|z_{1} / z_{0}\right| \geq K$.

Then, provided that $R_{0}>t$ is sufficiently large,

$$
\frac{2}{x} \geq \frac{e^{-x}}{x^{2}+y^{2}}(x \cos y-y \sin y) \geq \frac{1}{2 x} .
$$

Thus, by (5), if $R_{0}>t$ is sufficiently large, then

$$
|f(z)| \geq \lambda x \exp \left(\frac{1}{2 x}\right) \geq \frac{L}{x} \geq \frac{L}{|z|} .
$$

Similarly, if $R_{0}>t$ is sufficiently large, then

$$
\left|f\left(z_{0}\right)\right| \geq \lambda x_{0} \exp \left(\frac{1}{2 x_{0}}\right) \geq 2 L K \lambda x_{1} \exp \left(\frac{2}{x_{1}}\right) \geq L K\left|f\left(z_{1}\right)\right| .
$$

Case (2) is very similar, and is omitted. For case (3), suppose that $z=x+i y$, $z_{0}=x_{0}+i y_{0}$ and $z_{1}=x_{1}+i y_{1}$ are all points of $C_{n}\left(R_{0}\right)$, for some even $n \in \mathbb{N}$, with $\left|z_{1} / z_{0}\right| \geq K$. Recall that $|y-n \pi|<1 / 4$. Then, provided that $R_{0}>t$ is sufficiently large we can assume that $|x|$ is large compared to $y$, and so

$$
\frac{2 e^{-x}}{|x|} \geq \frac{e^{-x} \cos (y+\arctan (y / x))}{\sqrt{x^{2}+y^{2}}} \geq \frac{e^{-x}}{2|x|} .
$$

The remainder of the proof of this case is very similar to the remainder of the proof of case (1). Finally, case (4) is very similar to the proof of the case (3) and is omitted.

Our next two results are somewhat similar. They both say, roughly, that a suitable curve that does not meet $f^{-2}(H)$ must contain a subcurve which lies in the channels. Each result requires us to locate the preimages of $\mathbb{R}^{+}$, and so find the solutions to (6). We do this by estimating these solutions; first near the origin, and then for points of large, negative imaginary part.

Lemma 5.9. There exists $r_{0}>0$ and $L_{0}>1$ with the following property. Suppose that $R \geq r_{0}$, that $L \geq L_{0}$, that $K>1$, and that $z_{0}, z_{1} \in \mathbb{C}^{*} \backslash f^{-2}(H)$ are two points of modulus less than $1 / R$, such that $\left|z_{1} / z_{0}\right| \geq L K$. Suppose also that $\gamma \subset \mathbb{C}^{*} \backslash f^{-2}(H)$ is a curve from $z_{1}$ to $z_{0}$. Then there is a curve $\gamma^{\prime} \subset \gamma \cap C(R)$ joining endpoints $z_{0}^{\prime}$ and $z_{1}^{\prime}$, with $\left|z_{1}^{\prime} / z_{0}^{\prime}\right| \geq K$. More precisely, $\gamma^{\prime}$ lies in either $C^{+}(R)$ or $C^{-}(R)$.

Proof. Recall, from Observation 5.4 that $f^{-1}\left(\mathbb{R}^{+}\right) \subset f^{-2}(H)$. Recall also that if the point $x+i y \in f^{-1}\left(\mathbb{R}^{+}\right)$, then the pair $x, y$ is a solution to (6). We begin, therefore, by estimating the solutions to (6) near the origin; see Figure 6.

By the penultimate part of Lemma 5.5, the solutions to 6 near the origin do not cross the real axis. It follows that, near the origin, solutions to (6) can be written in the form

$$
\frac{e^{-x}}{x^{2}+y^{2}}(x \sin y+y \cos y)=n \pi+O(1), \quad \text { for } n \in \mathbb{Z} \text { as }|n| \rightarrow \infty,
$$




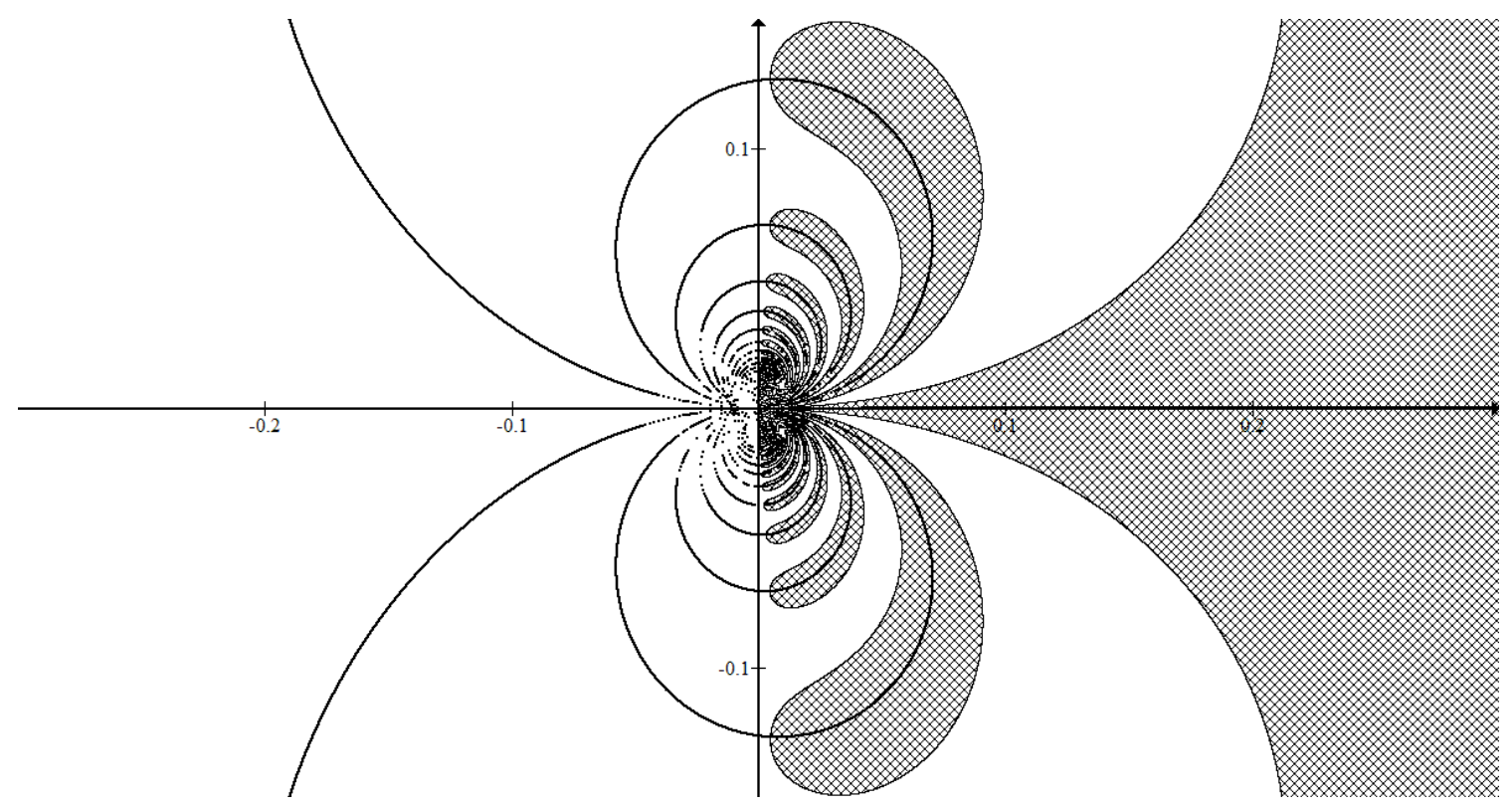

FIGURE 6 . In this figure the hatched area is $f_{32}^{-1}(H)$ and the black lines are the preimages of the positive real axis. Notice that, as calculated in Lemma 5.9, the preimages of the positive real axis are approximately circles. Note that there is some distortion near the origin.

where the $O(1)$ term is equal to $\arctan (y / x)$. By estimating the functions in (8) near the origin, we obtain that in a small neighbourhood of the origin

(9) $x^{2}+\left(y-\frac{1}{2 n \pi}\right)^{2}=\left(\frac{1}{2 n \pi}\right)^{2}+\left(x^{2}+y^{2}\right) \cdot O\left(\frac{1}{n}\right), \quad$ for $n \in \mathbb{Z}$ as $|n| \rightarrow \infty$.

Hence, when $|n|$ is large, the preimages of the positive real axis are close to circles $A_{n}$, where, for $n \in \mathbb{Z}, A_{n}$ is given by

$$
A_{n}:=\left\{z=x+i y \in \mathbb{C}: x^{2}+(y-p)^{2}=p^{2} \text { where } p=\frac{1}{2 n \pi}\right\} .
$$

Suppose that $z_{0}, z_{1}$ and $\gamma$ are as in the statement of the lemma. We can assume that these all lie in the upper half-plane. Recall that $\gamma$ cannot meet a preimage of the real line. Hence, if $R$ is sufficiently large, then $z_{0}$ and $z_{1}$ must lie in a domain which is very close to being the crescent between $A_{n}$ and $A_{n+1}$, for some large value of $n$.

It follows from (9) that all points in this crescent have modulus that is less than $\frac{1}{n \pi}+O\left(\frac{1}{n^{2}}\right)$. By a geometric calculation, we find that all points in this crescent of modulus less than $\frac{1}{n \pi \sqrt{17}}+O\left(\frac{1}{n^{2}}\right)$ lie in $C(R)$. (The constant 17 here follows from the fact that $\epsilon_{0}=1 / 4$.) The result then follows with $L_{0}=2 \sqrt{17}$.

The second lemma is similar in many respects to the first, though the proof is more complicated. 
Lemma 5.10. There exists $r_{\infty}>0$ and $L_{\infty}>1$ with the following property. Suppose that $R \geq r_{\infty}$, that $L \geq L_{\infty}$, that $K>1$, and that $z_{0}, z_{1} \in \mathbb{C}^{*} \backslash f^{-2}(H)$ are two points of modulus greater than $R$, such that $\left|z_{1} / z_{0}\right| \geq L K$. Suppose also that $\gamma \subset \mathbb{C}^{*} \backslash f^{-2}(H)$ is a curve from $z_{1}$ to $z_{0}$. Then there is a curve $\gamma^{\prime} \subset \gamma \cap C(R)$ joining endpoints $z_{0}^{\prime}$ and $z_{1}^{\prime}$, with $\left|z_{1}^{\prime} / z_{0}^{\prime}\right| \geq K$. More precisely, $\gamma^{\prime} \subset C_{n}(R)$ for some $n \in \mathbb{Z}$.

Proof. We begin by estimating solutions to (6) for points $z=x+i y$ where $|y / x|$ is small; see Figure 4. Observe that when this is the case, solutions to (6) can be written in the form

$$
\frac{e^{-x}}{x^{2}+y^{2}}(x \sin y+y \cos y)=n \pi+O(|y / x|), \quad \text { for } n \in \mathbb{Z}
$$

It follows by the compound angle formula that

$$
\sin (y+\arctan (y / x))=e^{x} \cdot \sqrt{x^{2}+y^{2}} \cdot(n \pi+O(|y / x|)), \quad \text { for } n \in \mathbb{Z} .
$$

Hence when $n$ is large compared to $|y / x|$, the preimages of the positive real axis are close to the sets $A_{n}^{\prime}$ where, for $n \in \mathbb{Z}$,

$$
A_{n}^{\prime}:=\left\{z=x+i y \in \mathbb{C}: \sin (y+\arctan (y / x))=e^{x} \cdot \sqrt{x^{2}+y^{2}} \cdot n \pi\right\} .
$$

When $n \neq 0$, no point on $A_{n}^{\prime}$ (and indeed no preimage of the real line) can meet the set of points that are solutions to (7), which we know are approximately the horizontal lines $\{z=x+i y \in \mathbb{C}: y=m \pi\}$, for some $m \in \mathbb{Z}$.

For all sufficiently large (and negative) values of $x$, the solution to $(10)$ is close to $y=m \pi$ for some $m \in \mathbb{Z}$. Note that points of large negative real part, and imaginary part close to an even multiple of $\pi$ map near the origin under $f$, and points of large negative real part, and imaginary part close to an odd multiple of $\pi$ map near infinity under $f$. We can deduce that $A_{n}^{\prime}$ is a curve that starts in the far left asymptotic to $y=m \pi$, travels some distance to the right, and then returns to the left asymptotic to $y=(m+1) \pi$. In particular, since $|\sin t| \leq 1$, for $t \in \mathbb{R}$, the rightmost point of $A_{n}^{\prime}$ is where

$$
e^{x} \cdot \sqrt{x^{2}+y^{2}} \cdot(n \pi+O(|y / x|))= \pm 1
$$

For large values of $|n|$, this gives $x=x_{n}=-\log |n|+O(\log \log |n|)$. Observe that there exists $\ell>0$, independent of $n$, such that for all sufficiently large values of $|n|$, if $x+i y \in A_{n}^{\prime}$ and $x<x_{n}-\ell$, then $x+i y \in C(R)$.

Suppose that $K, z_{0}, z_{1}$ and $\gamma$ are as in the statement of the lemma, and let $L>0$ be large. Let $\delta>0$ be small. It follows from Lemma 5.7 that if $L$ is sufficiently large, then there is a subcurve $\tilde{\gamma}$ of $\gamma$, which joins $z_{1}$ to a point $\tilde{z_{0}}$ with $\left|z_{1} / \tilde{z}_{0}\right| \geq L K / 2$, and such that if $z=x+i y \in \tilde{\gamma}$, then $|z|>R$ and $|y / x|<\delta$. We can assume that $\tilde{\gamma}$ lies in the upper half-plane.

Recall that $\tilde{\gamma}$ cannot meet the preimages of the positive real line. In the part of the plane where $\tilde{\gamma}$ lies we know that $|y / x|$ is small. So these points are close to the solutions to 10 . In other words $\tilde{\gamma}$ can be assumed to lie in the region very close to that between (in the obvious sense) $A_{n}^{\prime}$ and $A_{n+1}^{\prime}$, for some large value of $|n|$.

As noted earlier, all points in this region have real part less than

$$
x_{n}=-\log |n|+O(\log \log |n|) .
$$


All points in this region of real part less that $x_{n}-\ell$ lie in $C(R)$. The result of the lemma holds, for sufficiently large $r_{\infty}>0$, with (for example) $L_{\infty}=4(\ell+2 \pi)$.

We are now able to complete the proof, by showing that any component of $\mathbb{C}^{*} \backslash I$ is bounded in $\mathbb{C}^{*}$. To prove this, suppose by way of contradiction that $X$ is such a component that is not bounded in $\mathbb{C}^{*}$. Let $K$ be the constant from Lemma 5.8. First we choose

$$
L>\max \left\{2, L_{0}, L_{\infty}\right\}
$$

where $L_{0}$ is the constant in Lemma 5.9 and $L_{\infty}$ is the constant in Lemma 5.10. For this value of $L$, we let $R_{0}$ be the constant from Lemma 5.8. We then choose

$$
R>\max \left\{2, R_{0}, r_{0}, r_{\infty}\right\}
$$

where $r_{0}$ is the constant in Lemma 5.9 and $r_{\infty}$ is the constant in Lemma 5.10 .

For $\rho>1$, we use the notation

$$
D_{\rho}:=\left\{z \in \mathbb{C}^{*}:|z| \geq \rho \text { or }|z| \leq 1 / \rho\right\}
$$

When $\rho$ is large, points of $D_{\rho}$ have either large modulus or small modulus.

We construct sequences of points $\left(z_{k}^{0}\right)_{k \geq 0}$ and $\left(z_{k}^{1}\right)_{k \geq 0}$, and curves $\left(\gamma_{k}\right)_{k \geq 0}$, with $\gamma_{0} \subset X$, and with the following properties, for each $k \geq 0$ :

(I) $\gamma_{k} \subset C(R) \cap D_{2^{k} R}$ is a curve from $z_{k}^{0}$ to $z_{k}^{1}$.

(II) $\left|z_{k}^{1} / z_{k}^{0}\right| \geq K$.

(III) $\gamma_{k+1} \subset f\left(\gamma_{k}\right)$.

Note that (II) says that $\gamma_{k}$ is in one of the channels that make up $C(R)$ and, in addition, is either near the origin, or far from the origin (in which case it is also far to the left).

Note also that this construction completes the proof. To see this, observe that (III), together with Lemma 5.1, imply the existence of a point in $z \in \gamma_{0} \subset X$ which satisfies $f^{k}(z) \in \gamma_{k}$. This in turn implies, by (I), that $z \in I$, which is a contradiction.

The construction is by induction. The start of the induction is straightforward; it follows from Lemma 5.9 and Lemma 5.10, together with our assumption that $X$ is unbounded in $\mathbb{C}^{*}$, that we can choose $z_{0}^{0}, z_{0}^{1}$ and $\gamma_{0}$ with properties (I) and (II).

Now suppose that $k \geq 0$, and we have chosen $z_{k}^{0}, z_{k}^{1}$ and $\gamma_{k}$ with all the required properties. Set $\Gamma_{k}=f\left(\gamma_{k}\right)$. It follows from Lemma 5.8, and the fact that $L>2$, that $\Gamma_{k} \subset D_{2^{k}}$. Moreover, it is a consequence of Observation 5.3 that $\Gamma_{k}$ cannot meet $f^{-2}(H)$.

Since $\left|z_{k}^{1} / z_{k}^{0}\right| \geq K$, we have by Lemma 5.8 that

$$
\max \left\{\left|f\left(z_{k}^{1}\right) / f\left(z_{k}^{0}\right)\right|,\left|f\left(z_{k}^{0}\right) / f\left(z_{k}^{1}\right)\right|\right\} \geq L K .
$$

We then complete the construction with an application of either Lemma 5.9 if $\Gamma_{k} \subset\left\{z \in \mathbb{C}^{*}:|z| \leq 1 /\left(2^{k} R\right)\right\}$, or Lemma 5.10 if $\Gamma_{k} \subset\left\{z \in \mathbb{C}^{*}:|z| \geq 2^{k} R\right\}$. This completes the construction, and so completes the proof. 


\section{REFERENCES}

[Bak84] I. N. Baker, Wandering domains in the iteration of entire functions, Proc. Lond. Math. Soc. (3) 49 (1984), no. 3, 563-576.

[Bak87] I. N. Baker, Wandering domains for maps of the punctured plane, Ann. Acad. Sci. Fenn. Ser. A I Math. 12 (1987), no. 2, 191-198.

[BD98] I. N. Baker and P. Domínguez-Soto, Analytic self-maps of the punctured plane, Complex Variables Theory Appl. 37 (1998), no. 1-4, 67-91.

[Ber93] W. Bergweiler, Iteration of meromorphic functions, Bull. Amer. Math. Soc. 29 (1993), no. 2, 151-188.

[Ber95] W. Bergweiler, On the Julia set of analytic self-maps of the punctured plane, Analysis 15 (1995), no. 3, 251-256.

[Ber01] Walter Bergweiler, Singularities in Baker domains, Comput. Methods Funct. Theory 1 (2001), no. 1, 41-49.

[EMS19] Vasiliki Evdoridou, D. Martí-Pete, and David J Sixsmith, On the connectivity of the escaping set in the punctured plane, Preprint, arXiv:1908.07383v1 (2019).

[ER18] V. Evdoridou and L. Rempe-Gillen, Non-escaping endpoints do not explode, Bull. Lond. Math. Soc. 50 (2018), no. 5, 916-932.

[Ere89] A. E. Eremenko, On the iteration of entire functions, Dynamical systems and ergodic theory (Warsaw, 1986), Banach Center Publ., vol. 23, PWN, Warsaw, 1989, pp. 339-345.

[ES19] Vasiliki Evdoridou and David J Sixsmith, The topology of the set of non-escaping endpoints, International Mathematics Research Notices (2019).

[Evd16] V. Evdoridou, Fatou's web, Proc. Amer. Math. Soc. 144 (2016), no. 12, 5227-5240.

[FH06] Núria Fagella and Christian Henriksen, Deformation of entire functions with Baker domains, Discrete Contin. Dyn. Syst. 15 (2006), no. 2, 379-394.

[FM17] N. Fagella and D. Martí-Pete, Dynamic rays of bounded-type transcendental self-maps of the punctured plane, Discrete Contin. Dyn. Syst. Ser. A 37 (2017), 3123-3160.

[Kee88] Linda Keen, Dynamics of holomorphic self-maps of $\mathbb{C}^{*}$, Holomorphic functions and moduli, Vol. I (Berkeley, CA, 1986), Math. Sci. Res. Inst. Publ., vol. 10, Springer, New York, 1988, pp. $9-30$.

[Kön99] Harald König, Conformal conjugacies in Baker domains, J. Lond. Math. Soc. (2) 59 (1999), no. 1, 153-170.

[Kot87] J. Kotus, Iterated holomorphic maps on the punctured plane, Dynamical systems (Sopron, 1985), Lecture Notes in Econom. and Math. Systems, vol. 287, Springer, Berlin, 1987, pp. 10-28.

[Mak87] P. M. Makienko, Iterations of analytic functions in $\mathbf{C}^{*}$, Dokl. Akad. Nauk SSSR 297 (1987), no. 1, 35-37.

[Mak91] P. M. Makienko, Herman rings in the theory of iterations of endomorphisms of $\mathbf{C}^{*}$, Sibirsk. Mat. Zh. 32 (1991), no. 3, 97-103.

[Mar] D Martí-Pete, Escaping points and semiconjugation of holomorphic self-maps of the punctured plane, in preparation.

[Mar18] D. Martí-Pete, The escaping set of transcendental self-maps of the punctured plane, Ergod. Th. \& Dynam. Sys. 38 (2018), no. 2, 739-760.

[Mar19] D. Martí-Pete, Escaping Fatou components of transcendental self-maps of the punctured plane, to appear in Math. Proc. Camb. Phil. Soc., 2019.

[Osb13] J. W. Osborne, Spiders' webs and locally connected Julia sets of transcendental entire functions, Ergod. Th. \& Dynam. Sys. 33 (2013), no. 4, 1146-1161.

[Råd53] H. Rådström, On the iteration of analytic functions., Math. Scand. 1 (1953), 85-92.

[Rip08] P.J. Rippon, Baker domains, Transcendental Dynamics and Complex Analysis, London Math. Soc. Lecture Note Ser., vol. 348, Cambridge Univ. Press, Cambridge, 2008, pp. 371395. 
[RS09] P. J. Rippon and G. M. Stallard, Functions of small growth with no unbounded Fatou components, J. Anal. Math. 108 (2009), 61-86.

[RS11] P. J. Rippon and G. M. Stallard, Slow escaping points of meromorphic functions, Trans. Amer. Math. Soc. 363 (2011), no. 8, 4171-4201.

[RS12] P. J. Rippon and G. M. Stallard, Fast escaping points of entire functions, Proc. Lond. Math. Soc. (3) 105 (2012), no. 4, 787-820.

[RS13] P. J. Rippon and G. M. Stallard, Baker's conjecture and Eremenko's conjecture for functions with negative zeros, J. Anal. Math. 120 (2013), 291-309.

[Six11] D. J. Sixsmith, Entire functions for which the escaping set is a spider's web, Math. Proc. Cambridge Philos. Soc. 151 (2011), no. 3, 551-571.

[Six18] D. J. Sixsmith, Dynamical sets whose union with infinity is connected, Ergod. Th. \& Dynam. Sys. (2018), 110.

School of Mathematics and Statistics, The Open University, Milton Keynes MK7 $6 \mathrm{AA}, \mathrm{UK}$

(iD) https://orcid.org/0000-0002-5409-2663

E-mail address: vasiliki.evdoridou@open.ac.uk

Institute of Mathematics of the Polish Academy of Sciences, ul. Śniadeckich 8, 00-656 WARSAW, POLAND

(iD) https://orcid.org/0000-0002-0541-8364

E-mail address: dmartipete@impan.pl

Department of Mathematical Sciences, University of Liverpool, Liverpool L69 7ZL, UK

(iD) https://orcid.org/0000-0002-3543-6969

E-mail address: djs@liverpool.ac.uk 